\title{
Asymptotic states of accelerated qubits in nonzero background temperature
}

\author{
A. P. C. M Lima ${ }^{*}$, G. Alencar, and R. R. Landim $\odot$ \\ Departamento de Física, Universidade Federal do Ceará- Caixa Postal 6030, \\ Campus do Pici, 60455-760, Fortaleza, Ceará, Brazil
}

(Received 4 March 2020; accepted 28 May 2020; published 11 June 2020)

\begin{abstract}
The study of the Unruh effect naturally raises the interest for a deeper understanding of the analogy between temperature and acceleration. A recurring question is whether an accelerated frame can be distinguished from an inertial thermal bath in pure thermodynamic experiments; such problem has been approached in the literature and a consensus is yet to be fully reached. In the present work, we use the open quantum system formalism to investigate the case where both acceleration and background temperature are present. We find the asymptotic state density and entanglement generation from the Markovian evolution of accelerated qubits interacting with a thermal state of the external scalar field. Our results suggest that there is a very small asymmetry on the effects of the Unruh and background temperatures. Addressing the nonzero background temperature case is of both theoretical and phenomenological interest, thus the authors hope to enrich the existing discussions on the topic.
\end{abstract}

DOI: 10.1103/PhysRevD.101.125008

\section{INTRODUCTION}

The Minkowski vacuum, a Lorentz invariant, turns out to be seen as a Planckian thermal spectrum of particles in the frame of a uniformly accelerated observer; this is the widely known Unruh effect [1-3]. Being one of the key results from field quantization in general coordinates systems, still it arises in flat spacetime, without the need to include gravitational effects. The traditional treatment of the problem consists of considering a Unruh-Dewitt particle detector: a quantum particle with discrete nonrelativistic energy levels weakly linearly coupled to an external quantum field. The rate of excitation of the system when traveling in a hyperbolic trajectory is then calculated and shown to be proportional to a Planck factor. The generalization of this problem for finite background temperature, which will be of importance in the present work, has been worked on in Refs. [4,5].

A more recent point of view to the examination of Unruh thermalization has been addressed by considering a similar detector and field setup in the context of open quantum systems governed by a Markovian master equation [6]. This approach provides a richer analysis of the evolution of the system and the study of the phenomenon of entanglement generation for multiparticle detectors. Several generalizations

\footnotetext{
*augustopcml@gmail.com
}

Published by the American Physical Society under the terms of the Creative Commons Attribution 4.0 International license. Further distribution of this work must maintain attribution to the author(s) and the published article's title, journal citation, and DOI. Funded by SCOAP. to the problem have been proposed in the literature by considering different trajectories, finite distance between detectors, different fields, number of detectors, presence of boundaries, and others (Refs. [7-16]). The study of entanglement is a very rich subject and associating it with Unruh effect might provide us with further valuable insights. Also, linking the effect to another physical quantity may become an alternative for experimental observation, which is problematic due to the extremely high accelerations needed to induce actually detectable thermal spectra (see, however, Refs. [17,18]). As an example, in Ref. [19], it has been shown that entanglement can enhance the precision for Unruh thermalization detection.

A subject that has caught attention recently is the study of the apparent undistinguishability between the Unruh and thermal bath temperatures. For example, in Refs. [20-22], it is argued that some quantities such as density operator and detector response function are symmetric in background and Unruh temperatures, while [23] shows some aspects of distinguishability. Motivated by this problem, we propose to analyze the Unruh effect in the presence of nonzero thermal background temperature in the view of open quantum systems. We keep the analysis as simple as possible, by looking at the asymptotic states generated from the system evolution and comparing the dependency of the final states on both Unruh and background temperatures we mean to provide another test on the distinguishability of these effects. It is worth mentioning that distinct features between acceleration and thermalization have already been predicted using OQS formalism, such as in the case of the presence of reflecting boundaries [8] and two detectors with nonvanishing distance [7]. Thus, we focus on the 
simpler version (vanishing distance, no boundaries) of the setup in which the effects are originally undistinguished (when analyzed separately).

A second motivation, shared with similar works, comes from the fact that given the general smallness of the Unruh effect, background thermal effects are likely to be about as important as acceleration in the measures, and thus more realistic setup needs to include the inertial thermal bath. Then, by extending the analysis of [6] to nonzero background temperatures, we hope to provide further insight on the study of Unruh effect and the correlation between acceleration and temperature.

The rest of this paper is divided in three sections. In the next, we present a review of the formalism from [6], which we use as basis. In Sec. III, we expose our results for the case of finite temperatures. Section IV will consist of our final remarks on the problem.

\section{MARKOVIAN EVOLUTION OF DETECTORS IN MINKOWSKI VACUUM}

For the sake of completeness, we begin with a brief review of the method and results from [6], which we use as the start point for our proposed analysis in Sec. III. For a more detailed explanation, the reader can refer to the original reference.

The system in consideration is a two-level Unruh-Dewitt detector weakly coupled to an external massless scalar field. Instead of the standard transition rate calculation, the state density operator of the entire system (detector + field) is considered, and a reduced dynamics for the detector's state density is derived by a properly defined tracing operation over the field degrees of freedom.

A more interesting case happens when we consider two detectors following the same accelerated trajectory. As shown in [6], their final state may turn out to be entangled. This remarkable property opens a whole new window of possible discussions on the subject. As will be made clear, both the results for one or two detectors can be related to a common parameter which we will use as the key to our analysis in Sec. III.

\section{A. Time evolution for a single particle}

The total system Hamiltonian is

$$
H_{s}=H_{D}+H_{f}+H_{I}
$$

where $H_{D}$ and $H_{I}$ are, respectively, the detector and interaction Hamiltonians given by

$$
\begin{aligned}
H_{D} & =\frac{\omega}{2} \sigma_{z} \otimes \mathbf{1}, \\
H_{I} & =\lambda \sigma_{\mu} \otimes \Phi_{\mu},
\end{aligned}
$$

while $H_{f}$ is the usual free scalar field Hamiltonian. The field $\Phi_{\mu}$ appearing in the definition of $H_{I}$ is defined as

$$
\Phi_{\mu}=\chi_{\mu} \phi^{-}+\chi_{\mu}^{*} \phi^{+},
$$

where $\chi_{\mu}$ serve as generalized coupling constants, $\sigma_{0}$ is the identity $2 \times 2$ matrix, and $\sigma_{i}$ the Pauli matrices, $\phi^{ \pm}$are the positive and negative parts of the field expansion. For simplicity, these coupling constants will be assumed to satisfy

$$
\chi_{\mu} \chi_{\nu}=\delta_{\mu \nu} .
$$

The initial density operator of the system will be assumed to be

$$
\rho_{\text {Total }}(0)=\rho(0) \otimes \rho_{F},
$$

where $\rho$ and $\rho_{F}$ represent the densities of the detector and the field, respectively.

A properly defined tracing procedure over the field degrees of freedom leads to an expression for the time evolution of the detector's state density; however, it is a very complicated equation containing memory effects. By requiring that $\rho_{F}$ is a static configuration of the field over the time evolution generated by $H_{F}$ and the condition of weak coupling formally carried out at [24,25] (there are other assumptions, see [14] for a short explanation on the approximations considered and a more general alternative method to the one following), one obtains a Markovian (no memory effects) equation of motion, known as the Kossakowski-Lindblad equation,

$$
\dot{\rho}=-i\left[H_{\mathrm{eff}}, \rho(t)\right]+\mathcal{L}[\rho(t)]
$$

The operator $\mathcal{L}[\rho]$ in the above equation is called the Lindbladian, given by

$$
\begin{aligned}
\mathcal{L}[\rho] & =\frac{1}{2} \sum_{i, j=1}^{3} a_{i j}\left[2 \sigma_{j} \rho \sigma_{i}-\sigma_{i} \sigma_{j} \rho-\rho \sigma_{i} \sigma_{j}\right], \\
a_{i j} & =A \delta_{i j}-i B \epsilon_{i j k}+C n_{i} n_{j} .
\end{aligned}
$$

The coefficients $A, B$, and $C$ in the KossakowskiLindblad matrix $a_{i j}$ are given in terms of Fourier transforms of the field Wightman functions

$$
\begin{aligned}
\mathcal{G}(\omega) & =\int_{-\infty}^{\infty} d t \exp [i \omega t]\langle\phi[x(t)] \phi[x(0)]\rangle, \\
A & =\frac{1}{2}[\mathcal{G}(\omega)+\mathcal{G}(-\omega)], \\
B & =\frac{1}{2}[\mathcal{G}(\omega)+\mathcal{G}(-\omega)], \\
C & =\frac{1}{2}[\mathcal{G}(0)-\mathcal{G}(\omega)-\mathcal{G}(-\omega)]
\end{aligned}
$$


and

$$
\begin{aligned}
H_{\text {eff }} & =\frac{\Omega}{2} \vec{n} \cdot \vec{\sigma}, \\
\Omega & =\omega+i[\mathcal{K}(-\omega)-\mathcal{K}(\omega)], \\
\mathcal{K}(\omega) & =\frac{1}{i \pi} P \int_{-\infty}^{\infty} d \lambda \frac{\mathcal{G}(\lambda)}{\lambda-\omega},
\end{aligned}
$$

with $P$ denoting the principal value. The $\mathcal{K}$ terms in the effective Hamiltonian $H_{\text {eff }}$ represent the well-known Lamb shift, a renormalization process is supposed to take care of divergences. As we will see, these terms do not appear in the final asymptotic states so we will not discuss them further.

When $\rho_{F}$ is Minkowski's vacuum, we have

$$
\begin{aligned}
\langle\phi(x+\Delta x) \phi(x)\rangle & =G(x+\Delta x, x) \\
& =-\frac{1}{4 \pi^{2}} \frac{1}{\left(\Delta x^{0}-i \epsilon\right)^{2}-\overrightarrow{\Delta x^{2}}} .
\end{aligned}
$$

The detector is moving on a hyperbolic trajectory with proper time $t$,

$$
\begin{aligned}
x^{0}(t) & =\frac{1}{a} \sinh (a t), \\
x^{1}(t) & =\frac{1}{a} \cosh (a t), \\
x^{2} & =x^{3}=0 .
\end{aligned}
$$

Inserting this trajectory in (10) and integrating in (8), we obtain

$$
\begin{aligned}
\mathcal{G}(\omega) & =\frac{1}{2 \pi} \frac{\omega}{1-e^{-\beta_{U} \omega}}, \\
A & =\frac{\omega}{4 \pi}\left(\frac{1+e^{-\beta_{U} \omega}}{1-e^{-\beta_{U} \omega}}\right), \\
B & =\frac{\omega}{4 \pi}, \\
C & =\frac{\omega}{4 \pi}\left(\frac{2}{\beta_{U} \omega}-\frac{1+e^{-\beta_{U} \omega}}{1-e^{-\beta_{U} \omega}}\right) .
\end{aligned}
$$

By plugging these back in (7) and conveniently decomposing the state density operator as

$$
\rho=\frac{1}{2}\left(1+\sum_{i} \rho_{i} \sigma_{i}\right)
$$

we can express it as a vector $\left|\rho_{i}\right\rangle$ known as the Bloch vector. Thus, one obtains the time evolution for its components,

$$
\frac{\partial}{\partial t}|\rho(t)\rangle=-2 \mathcal{H}|\rho(t)\rangle+|\eta\rangle,
$$

where $\eta_{i}=-4 B n_{i}$ and

$$
\mathcal{H}=\left(\begin{array}{ccc}
a & b+\Omega_{3} & c-\Omega_{2} \\
b-\Omega_{3} & \alpha & \beta+\Omega_{1} \\
c+\Omega_{2} & \beta-\Omega_{1} & \gamma
\end{array}\right),
$$

where

$$
\begin{aligned}
a & =2 A+C\left(n_{2}^{2}+n_{3}^{2}\right), & b & =-C n_{1} n_{2}, \\
\alpha & =2 A+C\left(n_{1}^{2}+n_{3}^{2}\right), & & c=-C n_{1} n_{3}, \\
\gamma & =2 A+C\left(n_{1}^{2}+n_{2}^{2}\right), & & \beta=-C n_{2} n_{3}, \\
\Omega_{i} & =\frac{\Omega}{2} n_{i}, \quad n=1,2,3 . & &
\end{aligned}
$$

The solution of Eq. (14) allows one to compute, for example, the usual response function for the two-level Unruh-Dewitt detector. Here, for simplicity and conciseness, we will focus only on the asymptotic state $(t \rightarrow \infty)$,

$$
\rho_{i}=-\frac{B}{A} n_{i}=\tanh \left(\frac{\beta_{U} \omega}{2}\right) n_{i} .
$$

This state density can be identified with the one for thermal equilibrium in a $1 / \beta_{U}$ temperature heat bath,

$$
\rho_{\beta}=\frac{e^{-i \beta_{U} H_{D}}}{\operatorname{Tr}\left[e^{-i \beta_{U} H_{D}}\right]} .
$$

This indicates that the accelerated particle behaves as if it was immersed in a thermal bath of temperature $a /(2 \pi)$ (i.e., the Unruh effect) and achieves thermal equilibrium at asymptotically high proper times.

\section{B. Two particles and entanglement generation}

For the case of two particles following the same trajectory (11), the system will also be described by an equation of the type (6), with the Lindbladian given by

$$
\begin{aligned}
\mathcal{L}[\rho]= & \sum_{i, j} a_{i j}\left(\left[\left(\sigma_{j} \otimes \sigma_{0}\right) \rho\left(\sigma_{i} \otimes \sigma_{0}\right)-\frac{1}{2}\left\{\sigma_{i} \sigma_{j} \otimes \sigma_{0}, \rho\right\}\right]\right. \\
& +\left[\left(\sigma_{0} \otimes \sigma_{j}\right) \rho\left(\sigma_{0} \otimes \sigma_{i}\right)-\frac{1}{2}\left\{\sigma_{0} \otimes \sigma_{i} \sigma_{j}, \rho\right\}\right] \\
& +\left[\left(\sigma_{j} \otimes \sigma_{0}\right) \rho\left(\sigma_{0} \otimes \sigma_{i}\right)-\frac{1}{2}\left\{\sigma_{i} \otimes \sigma_{j}, \rho\right\}\right] \\
& \left.+\left[\left(\sigma_{0} \otimes \sigma_{j}\right) \rho\left(\sigma_{i} \otimes \sigma_{0}\right)-\frac{1}{2}\left\{\sigma_{j} \otimes \sigma_{i}, \rho\right\}\right]\right) .
\end{aligned}
$$

The $a_{i j}$ coefficients are the same as in the single particle case and the new effective Hamiltonian is given by 


$$
H_{\mathrm{eff}}=H_{\mathrm{eff}}^{(1)}+H_{\mathrm{eff}}^{(2)}+H_{\mathrm{eff}}^{(1,2)}
$$

where $H_{\mathrm{eff}}^{(1)}$ and $H_{\mathrm{eff}}^{(2)}$ are defined analogously to (9b) and

$$
\begin{aligned}
H_{\mathrm{eff}}^{(1,2)}= & i \sum_{i j}\left\{[\kappa(\omega)+\kappa(-\omega)] \delta_{i j}\right. \\
& \left.+[\kappa(0)-\kappa(\omega)-\kappa(-\omega)] n_{i} n_{j}\right\} .
\end{aligned}
$$

The term above represents an indirect interaction; however, from the definition $(9 \mathrm{c})$, we see that $\kappa(\omega)$ is an odd function and thus the term cancels out, leaving just the individual and acceleration independent Lamb shifts. For further simplification on the analysis of the acceleration induced effects, the effective Hamiltonian will be dropped from (6) in this case.

Decomposing the reduced density as

$$
\begin{aligned}
\rho(t)= & \frac{1}{4}\left[\sigma_{0} \otimes \sigma_{0}+\sum_{i} \rho_{0 i}(t) \sigma_{0} \otimes \sigma_{i}\right. \\
& \left.+\sum_{i} \rho_{i 0}(t) \sigma_{i} \otimes \sigma_{0}+\sum_{i, j} \rho_{i j}(t) \sigma_{i} \otimes \sigma_{j}\right],
\end{aligned}
$$

the following equations are obtained:

$$
\begin{aligned}
\dot{\rho}_{0 i}= & -4 A \rho_{0 i}+2 B(2+T) n_{i}-2 B \sum_{k} n_{k} \rho_{i k}, \\
\dot{\rho}_{i 0}= & -4 A \rho_{i 0}+2 B(2+T) n_{i}-2 B \sum_{k} n_{k} \rho_{k i}, \\
\dot{\rho}_{i j}= & -4 A\left[2 \rho_{i j}+\rho_{j i}-T \delta_{i j}\right], \\
& +2 B\left[n_{i}\left(2 \rho_{0 j}+\rho_{j 0}\right)+n_{j}\left(2 \rho_{i 0}+\rho_{0 i}\right)\right. \\
& -\delta_{i j} \sum_{k} n_{k}\left(\rho\left(\rho_{k 0}+\rho_{0 k}\right)\right],
\end{aligned}
$$

which in the asymptotic state reduce to

$$
\begin{aligned}
& \hat{\rho}_{i 0}=\hat{\rho}_{0 i}=\frac{R(T+3)}{3+R^{2}} n_{i}, \\
& \hat{\rho}_{i j}=\hat{\rho}_{j i}=\frac{1}{3+R^{2}}\left[n_{i} n_{j}(T+3)+\left(T-R^{2}\right) \delta_{i j}\right],
\end{aligned}
$$

where $R=B / A$ and $T=\sum_{i} \rho_{i i}$ (which is constant of movement, as can be checked by taking the trace in the above equations for $\rho_{i j}$ ). Those satisfy $0 \leq R \leq 1$ and $-3 \leq T \leq 1$ as requirement of the density operator being strictly positive. Notice that the quantity $\mathrm{R}$ also appears at the single particle final state (17).

To analyze the entangling of the two qubits, one can compute the concurrence [26-28]. Let $\rho_{c}$ be defined by

$$
\rho_{C}=\rho\left(\sigma_{2} \otimes \sigma_{2}\right) \rho^{T}\left(\sigma_{2} \otimes \sigma_{2}\right),
$$

with positive eigenvalues $\lambda_{1,2,3,4}$; the concurrence $\mathcal{C}$ is then given by

$$
\mathcal{C}[\rho]=\max \left\{\lambda_{1}-\lambda_{2}-\lambda_{3}-\lambda_{4}, 0\right\}
$$

assuming values $0 \leq \mathcal{C} \leq 1$, with unity value representing a completely entangled state and zero a completely untangled one.

The concurrence for the asymptotic configuration in (24) is given by

$$
\mathcal{C}[\hat{\rho}]=\frac{3-R^{2}}{2\left(3+R^{2}\right)}\left[\frac{5 R^{2}-3}{3-R^{2}}-T\right],
$$

for $T<\left(5 R^{2}-3\right) /\left(3-R^{2}\right)$, or zero otherwise.

As a simple example, one can consider the two detectors prepared in an originally separable state $(\mathcal{C}=0)$,

$$
\rho(0)=(1+\vec{p} \cdot \vec{\sigma}) \otimes(1+\vec{q} \cdot \vec{\sigma}),
$$

with $\vec{q}$ and $\vec{p}$ denoting unit vectors. Then, the maximum entanglement in the final state will be achieved for $T=$ $\vec{q} \cdot \vec{p}=-1$ and will be given by

$$
\mathcal{C}[\hat{\rho}]=\frac{2 R^{2}}{3+R^{2}},
$$

illustrating thus, the entanglement generation for two initially untangled atoms and its dependency on the acceleration. The results reviewed in this section are identical to those one obtains from considering static particles in a common thermal bath.

This concludes our review from [6]. Next, we want to analyze the case where besides an acceleration, there is also a finite background temperature. Our main focus will be the question as to whether the effects induced by acceleration and temperature are distinguishable, and if so, how different these effects are.

\section{FINITE TEMPERATURE BACKGROUND}

It has been shown in earlier works that under certain circumstances uniform acceleration will have distinct features from an inertial thermal bath in the state density evolution of the detectors, such as the presence of reflecting boundaries and finite distance between detectors $[7,8]$. Instead, here we consider the simpler case reviewed in the last section and introduce a background uniform temperature as measured by an inertial observer. We want to compare, in the case where both effects are present, the temperature and acceleration dependencies of the process, as well as having a look at the summed effect. 
The initial configuration of the field is given by

$$
\rho_{f}(0)=\frac{e^{-\beta H_{f}}}{\operatorname{Tr}\left[e^{-\beta H_{f}}\right]}
$$

which is an equilibrium state of the field's natural evolution $\left[\rho_{f}(0), H_{f}\right]=0$. In this configuration, the correlation function for the field is

$G^{+}(x+\Delta x, x)=-\frac{1}{4 \beta} \sum_{n=-\infty}^{\infty} \frac{1}{\left(\Delta x^{0}-i \epsilon\right)^{2}-\overrightarrow{\Delta x}^{2}+i n \beta}$.

For a static trajectory, it reduces to the same expression as in the accelerated case with $2 \pi \beta \rightarrow a$. The explicit form for a trajectory of type (11) is worked out in [5] (se also the explicit form in [29]); for the case $\Delta x=x(t)-x(0)$, it reduces to

$$
\begin{aligned}
G^{+}(t, 0)= & \frac{1}{8 \beta \alpha} \operatorname{csch}^{2}\left(\frac{\pi t}{\alpha}\right)\left\{\operatorname{coth}\left[\frac{\alpha}{\beta}\left(e^{2 \pi t / \alpha}-1\right)\right]\right. \\
& \left.-\operatorname{coth}\left[\frac{\alpha}{\beta}\left(1-e^{-2 \pi t / \alpha}\right)\right]\right\},
\end{aligned}
$$

where $\alpha$ and $\beta$ are the inverse of the Unruh and background temperatures, respectively, and the $i \epsilon$ factors have been suppressed.

Unfortunately, the Fourier transform for expression (32) does not seen to have a closed expression. We employ then a trick from [5], by separating a background temperature "correction,"

$$
G^{+}(t, 0)=-\frac{1}{4 \alpha^{2}} \operatorname{csch}^{2}(\pi t / \alpha)+\Delta G_{\alpha \beta}^{+},
$$

where

$$
\Delta G_{\alpha \beta}^{+}=\frac{1}{4 \alpha^{2}} \operatorname{csch}^{2}(\pi t / \alpha)+G^{+} .
$$

This simply factors out the pure acceleration part which we already know how to treat. The remaining contribution $\Delta G_{\alpha \beta}^{+}$is well behaved (on the sense that its singularity at $t=0$ is removable) on the real axis, and thus we can drop the $i \epsilon$ factors and easily integrate it numerically (see the plots in Fig. 1). Equivalently, one could instead separate the pure temperature term and deal with the remaining part [see (38)].

From the expressions for the asymptotic state densities (17), (24), we can narrow the analysis to the examination of the quantity $R=B / A$. Conveniently, $G_{\beta}^{+}(t)$ is an even function of $t$, so that $B(8 \mathrm{c})$ remains unchanged. For $A(8 \mathrm{~b})$, we have

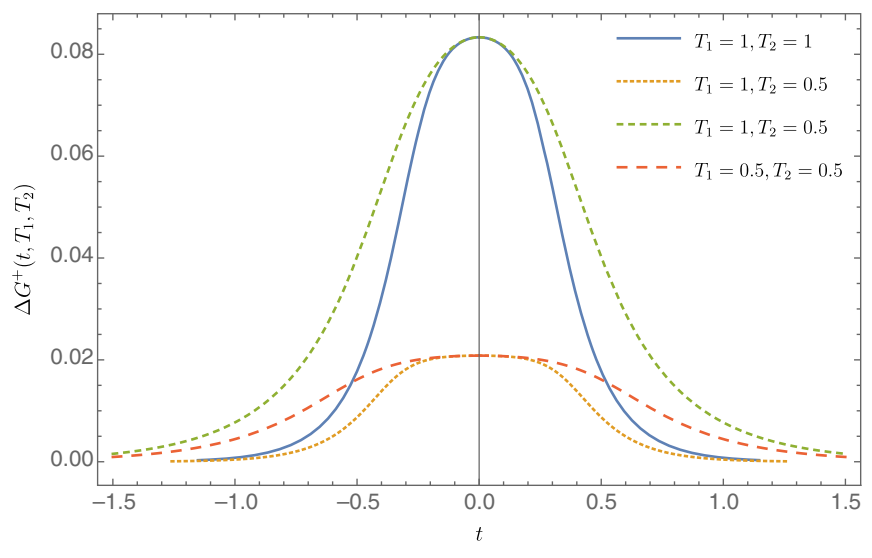

FIG. 1. Plots of $\Delta G_{\beta}^{+}\left(t, T_{1}, T_{2}\right)$. The curves slightly resemble a Gaussian, with peak value $T_{1}^{2} / 12$ at $t=0$. Notice that for same $T_{1}$, the curve characteristic width will be decreasing with $T_{1}$.

$$
A \rightarrow A+\int_{-\infty}^{\infty} d t \cos (\omega t) \Delta G_{\alpha \beta}^{+}
$$

Then, let the quantity $\gamma(\omega, \alpha, \beta)$ be defined by

$$
\gamma(\omega, \alpha, \beta)=\int_{-\infty}^{\infty} d t \cos (\omega t) \Delta G_{\alpha \beta}^{+},
$$

so $R$ can be written as

$R\left(\omega, \beta^{-1}, \alpha^{-1}\right)=\left[\operatorname{coth}\left(\frac{\alpha \omega}{2}\right)+\frac{4 \pi}{\omega} \gamma(\omega, \alpha, \beta)\right]^{-1}$.

From Eq. (32), one would guess acceleration and background temperature have very distinguishable effects; however, as we will see shortly, the asymmetry of $R$ in the two temperatures is generally very small.

Our main focus will be the analysis of general features, so we will be using simplistic values as the arguments of (37) rather than more experimentally realistic ones (see Sec. III A). First, let us have a look at the acceleration dependency of $R$ for a few different background temperatures [Fig. 2(a)]. It can be seen that the values decrease progressively with background temperature for small accelerations.

Next, we plot the values of $R$ for different detector frequency values. The curves in Fig. 2(b) suggest a monotonic dependency; for smaller values of $\omega$, we have an approximately linear growth. Now to check what happens when we swap the background and Unruh temperatures, the comparative curves are plotted in Fig. 3(a). As mentioned before, just by looking at the Green's function (32), one could be naively led to think there would be a big difference on the values of $\mathrm{R}$ obtained by swapping $\alpha$ for $\beta$. In Fig. 3(b), we can observe the values of $s\left(\omega, T_{1}, T_{2}\right)=$ $R\left(\omega, T_{1}, T_{2}\right)-R\left(\omega, T_{2}, T_{1}\right)$.

Notice that the equivalent acceleration/temperature curves have very little deviations from one another. 


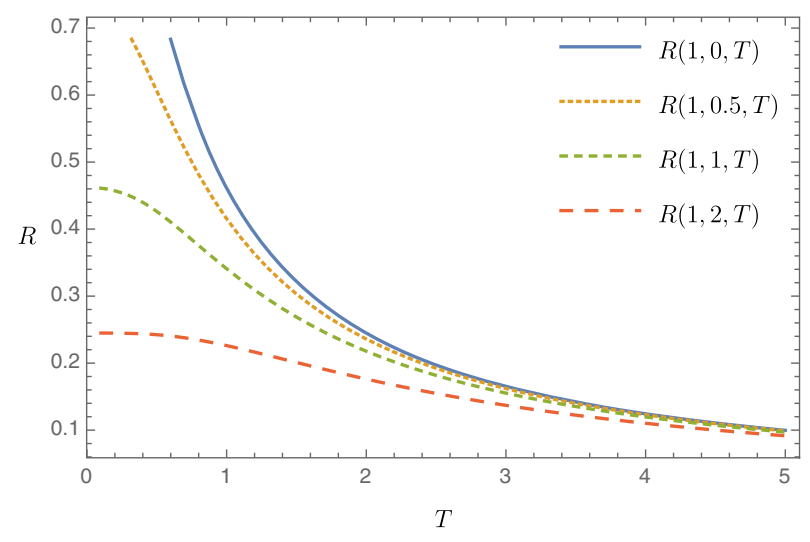

(a)

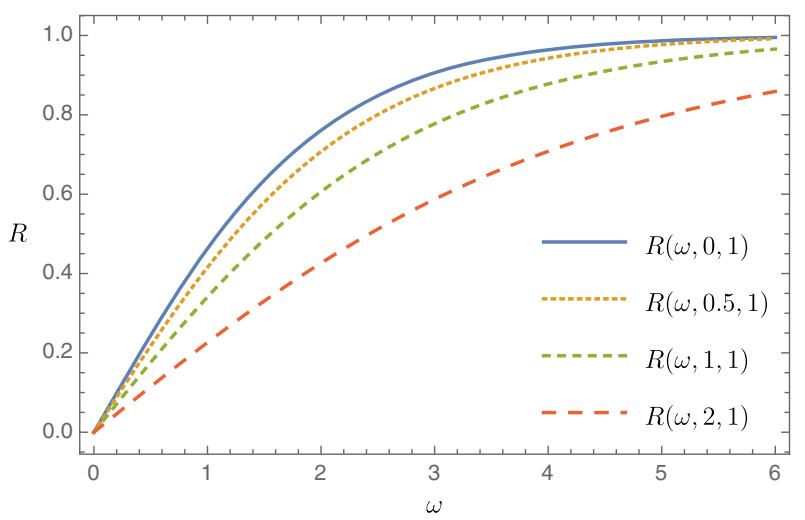

(b)

FIG. 2. Plotting $R\left(\omega, T_{1}, T_{2}\right)$ against temperature and detector frequency. (a) Plot of $R\left(1, T^{\prime}, T\right)$ against $T$ for a few values of $T^{\prime}$. (b) Plot of $R\left(\omega, T, T^{\prime}\right)$ against $\omega$ for a few values of $T^{\prime}$.

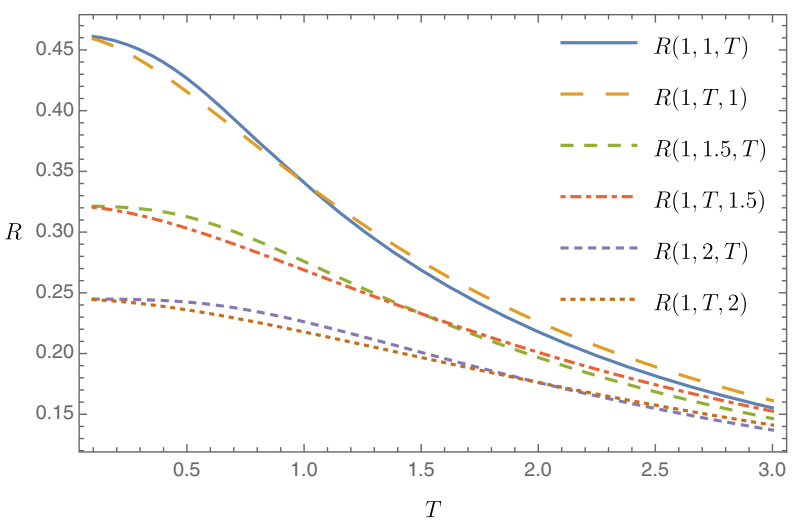

(a)

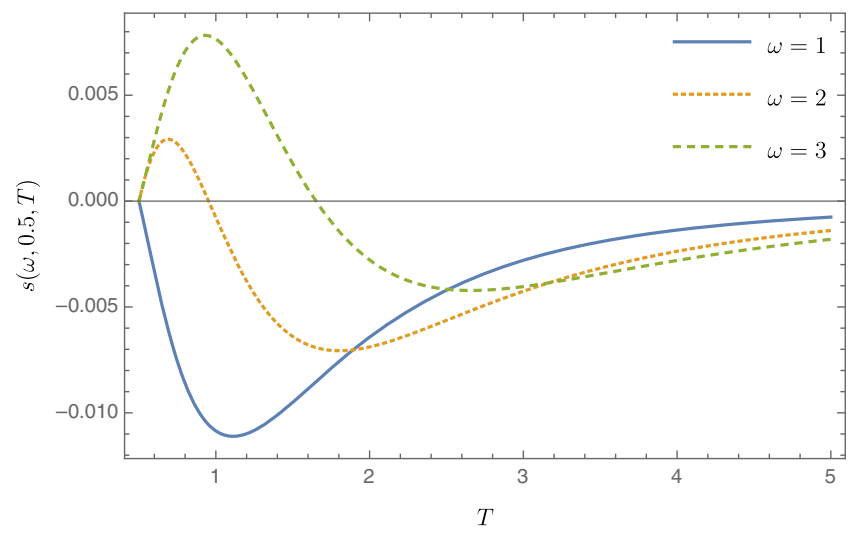

(b)

FIG. 3. Asymmetry of $R$ in the Unruh and background temperatures. (a) Values of $R\left(1, T, T^{\prime}\right)$ and $R\left(1, T^{\prime}, T\right)$ for a few examples of $\left(T, T^{\prime}\right)$. (b) Values of $s(\omega, 0.5, T)=R(\omega, 0.5, T)-R(\omega, T, 0.5)$ for $\omega=1,2,3$.

By analyzing the temperature and frequency dependencies, we can infer that this "closeness" feature is quite general. First, suppose we tinker with the frequency parameter, notice that for high frequency values, $R$ asymptotically approaches unit value, and for low frequencies the values vary almost linearly [Fig. 2(b)], thus generating effects almost proportional to those in Fig. 3(a). Now, we manipulate temperatures, if Unruh and background temperature are too different, say $T \gg T^{\prime}$, then $R\left(\omega, T^{\prime}, T\right)$ will be close to $R(\omega, 0, T)$ and thus there will not be a much bigger distinguishability either. Last, changing both temperatures proportionally can be equivalently done by switching time scale and changing frequency instead, so it falls back to the $\omega$ analysis (see Sec. III A).

\section{A. Discussing realistic values}

Up to now we have only used rather fictitious values for the parameters $\omega, \alpha$, and $\beta$, ignoring the magnitude order at which these quantities might seen plausible to consider.
This approach has been used to facilitate our analysis by generating more sensible results.

To get a look at more realistic values, we need to restore the constants involved. First, the frequency values, for a two-level system with energy gap $E$, we will have $\omega=E / \hbar$. Thus, for a frequency of say, $1 \mathrm{eV}$, the frequency time argument will be of $10^{15}$ order. It is known that very high accelerations are needed to generate even a seemingly small Unruh temperature; however, let us assume the acceleration induced thermalization stands on the same, or close to the order of a plausible background temperature as to generate a measurable effect. The general time coefficient $k_{B} T / \hbar$ in the arguments of the Green's function will be of order $10^{11} \times O[T]$.

From the "thermal correction" part of the Green's function (34), we can see that the detector only effectively interacts with the background temperature for a short time period (this is not really accurate as this interaction is not factored out, but forcefully separated in the expression), the 
width of the interval is proportional to the acceleration. Also, the usual part of Unruh thermalization has the argument proportional to $\omega / a$ (or $\omega / T$ ). Then, we are able to simplify the arguments in (37) by adjusting the units of the usual time scales. In other words, we have $R\left(\omega, T, T^{\prime}\right)=$ $R\left(\lambda \omega, \lambda T, \lambda T^{\prime}\right)$ for any positive $\lambda$, as can be easily seen from (34), (37). The close to unity values used earlier correspond to $\alpha \sim \beta$ and $\beta \omega \sim k_{B}$; this is achievable for a temperature of $1 K$ using energy gaps on the hydrogen fine structure order. Unfortunately, the acceleration values corresponding to this temperature are still too high, and a direct observation in the present case would require a much more sensible (low energy) detector. A more realistic option to achieve sensible values would be to consider circular trajectories (see $[5,16]$ ), which might be the subject for a more phenomenology oriented work in the near future. As shown in $[17,18]$, the Unruh effect in this case can be related to Sokolov-Ternov terms, which are experimentally observed.

Usually, it would be reasonable to expect background temperature values to be higher than the acceleration induced effect. In this case, it would be most efficient to separate the field Green's function as

$$
G^{+}=-\frac{1}{4 \beta^{2}} \operatorname{csch}^{2}(\pi t / \beta)+\Delta G_{\beta \alpha}^{+},
$$

where $\Delta G_{\beta \alpha}^{+}$is defined in analogy to (34). This way, we generate a small acceleration "correction" to the inertial thermalization. This is advantageous because in the (33) case, the width of the usual time scale is increasing in $\alpha$, whereas in the above case, it increases with $\beta$, making it easier to do precise integrations by picking whichever factor has a higher value.

\section{FINAL REMARKS}

In the present work, we proposed an extension to the problem presented in [6] by inserting a nonzero background temperature on the analysis of Unruh-Dewitt detectors as open quantum systems. We proceeded by keeping the calculations as simple as possible, looking at the asymptotic states obtained from a Markovian evolution of the particle detectors. As we did not find a closed expression for the Fourier transform of the external field correlations, the results are obtained numerically (Figs. 2 and 3). Also, we used these results as a further test to the acceleration-temperature correspondence.
The presence of a background temperature, as expected, contributed to further decrease entanglement generation between detectors, and its temperature/acceleration evolution curves look pretty familiar to the usual Unruh effect. On testing whether the asymptotic states were symmetrical in exchanging background and Unruh temperatures, we found that there is a small difference in the plotted curves, further suggesting that a common thermal bath and the acceleration induced one have distinct, although very close, properties. Since the analysis provided is pure Markovian, including memory effects and finite time behaviors might be of key importance and are to be approached in future works. Besides the close analogy between Unruh effect and thermal baths, the authors do not know why the corresponding curves found in Fig. 3(a) are so proximate to each other. Further works with different approximations in this scenario, such as non-Markovian calculations might show different results.

The acceleration needed for the Unruh effect to be actually measurable is unfortunately too high to achieve with current technology (at least in rectilinear trajectory). A reasonable work around would be to study the analogous case of the particle detector in a circular uniform motion (an open quantum system approach to Unruh effect in circular trajectory can be found in [16]; see also [17,18] for discussion on experimental detectability). But even in very high acceleration scenarios where the Unruh thermalization could be experimentally observed, one could expect background temperature effects to be of comparable or higher order. Thus, they should be accounted for in further works that propose to tackle a more realistic view of the problem.

Although the analysis provided is very simplified and the parameters used are rather unrealistic in a experimental perspective, the authors hope to catch some key features of the phenomena and that the results proposed here might provide insights for further works to come.

\section{ACKNOWLEDGMENTS}

The authors would like to thank Alexandra Elbakyan and sci-hub for removing all barriers in the way of science. We acknowledge the financial support by Conselho Nacional de Desenvolvimento Científico e Tecnológico (CNPq) and Fundação Cearense de Apoio ao Desenvolvimento Científico e Tecnológico (FUNCAP) through PRONEM PNE0112-00085.01.00/16. 
[1] W. G. Unruh, Phys. Rev. D 14, 870 (1976).

[2] P. C. W. Davies, J. Phys. A 8, 609 (1975).

[3] L. C. B. Crispino, A. Higuchi, and G. E. A. Matsas, Rev. Mod. Phys. 80, 787 (2008).

[4] T. Padmanabhan and T. P. Singh, Phys. Rev. D 38, 2457 (1988).

[5] S. S. Costa and G. E. A. Matsas, Phys. Rev. D 52, 3466 (1995).

[6] F. Benatti and R. Floreanini, Phys. Rev. A 70, 012112 (2004).

[7] J. Hu and H. Yu, Phys. Rev. A 91, 012327 (2015).

[8] J. Zhang and H. W. Yu, Phys. Rev. D 75, 104014 (2007).

[9] J. Zhang and H. W. Yu, Phys. Rev. A 75, 012101 (2007).

[10] Y. Yang, J. Hu, and H. Yu, Phys. Rev. A 94, 032337 (2016).

[11] D. Moustos and C. Anastopoulos, Phys. Rev. D 95, 025020 (2017).

[12] J. Hu and H. Yu, Phys. Rev. D 88, 104003 (2013).

[13] G. Menezes, N. F. Svaiter, and C. A. D. Zarro, Phys. Rev. A 96, 062119 (2017).

[14] B. Sokolov, J. Louko, S. Maniscalco, and I. Vilja, Phys. Rev. D 101, 024047 (2020).

[15] J. Doukas and B. Carson, Phys. Rev. A 81, 062320 (2010).
[16] J. She, J. Hu, and H. Yu, Phys. Rev. D 99, 105009 (2019).

[17] E. T. Akhmedov and D. Singleton, Int. J. Mod. Phys. A 22, 4797 (2007).

[18] E. T. Akhmedov and D. Singleton, JETP Lett. 86, 615 (2008).

[19] Z. Tian, J. Wang, J. Jing, and A. Dragan, Ann. Phys. (Amsterdam) 377, 1 (2017).

[20] S. Kolekar and T. Padmanabhan, Phys. Rev. D 89, 064055 (2014).

[21] S. Kolekar, Phys. Rev. D 89, 044036 (2014).

[22] S. Kolekar and T. Padmanabhan, Classical Quantum Gravity 32, 202001 (2015).

[23] C. Chowdhury, S. Das, S. Dalui, and B. R. Majhi, Phys. Rev. D 99, 045021 (2019).

[24] E. B. Davies, Commun. Math. Phys. 39, 91 (1974).

[25] E. B. Davies, Math. Ann. 219, 147 (1976).

[26] S. Hill and W. K. Wootters, Phys. Rev. Lett. 78, 5022 (1997).

[27] W. K. Wootters, Phys. Rev. Lett. 80, 2245 (1998).

[28] W. K. Wootters, Quantum Inf. Comput. 1, 27 (2001).

[29] H. A. Weldon, Phys. Rev. D 62, 056010 (2000). 